\title{
Étude de la complexité d'un événement : les responsables politiques québécois et les immigrants illégaux haïtiens, 1972-1974
}

\section{Martin Pâquet et Érick Duchesne}

Volume 50, numéro 2, automne 1996

URI : https://id.erudit.org/iderudit/305507ar

DOI : https://doi.org/10.7202/305507ar

Aller au sommaire du numéro

Éditeur(s)

Institut d'histoire de l'Amérique française

ISSN

0035-2357 (imprimé)

1492-1383 (numérique)

Découvrir la revue

Citer cet article

Pâquet, M. \& Duchesne, É. (1996). Étude de la complexité d’un événement : les responsables politiques québécois et les immigrants illégaux haïtiens, 1972-1974. Revue d'histoire de l'Amérique française, 50(2), 173-200. https://doi.org/10.7202/305507ar

\section{Résumé de l'article}

D'octobre 1972 à novembre 1974, une affaire bouleverse l'échiquier politique québécois, celle du sort réservé à de nombreux ressortissants haïtiens considérés comme immigrants illégaux par Ottawa. À travers cette étude de cas, cet article discerne, en suivant des principes dialogique et synchronique d'analyse, les différentes facettes du changement social ainsi que ses dynamiques structurelles et conjoncturelles, présentes au Québec des années 1970. En s'inspirant des approches interprétatives de la microstoria, il offre une reconstruction des modes de rationalité de l'événement, révélant ainsi toute sa complexité inhérente. Enfin, il propose une redéfinition des notions d'événement, de structure et de conjoncture.
Tous droits réservés @ Institut d'histoire de l'Amérique française, 1996
Ce document est protégé par la loi sur le droit d'auteur. L’utilisation des services d’Érudit (y compris la reproduction) est assujettie à sa politique d'utilisation que vous pouvez consulter en ligne.

https://apropos.erudit.org/fr/usagers/politique-dutilisation/ 


\title{
ÉTUDE DE LA COMPLEXITÉ D'UN ÉVÉNEMENT LES RESPONSABLES POLITIQUES QUÉBÉCOIS ET LES IMMIGRANTS ILLÉGAUX HAÏTIENS 1972-1974
}

\author{
MARTIN PÂQUET \\ Université York \\ Collège universitaire Glendon \\ ÉRICK DUCHESNE \\ Université de l'Alberta \\ Faculté Saint-Jean
}

\section{RÉSUMÉ}

D'octobre 1972 à novembre 1974, une affaire bouleverse l'échiquier politique québécois, celle du sort réservé à de nombreux ressortissants haïtiens considérés comme immigrants illégaux par Ottawa. À travers cette étude de cas, cet article discerne, en suivant des principes dialogique et synchronique d'analyse, les différentes facettes du changement social ainsi que ses dynamiques structurelles et conjoncturelles, présentes au Québec des années 1970. En s'inspirant des approches interprétatives de la microstoria, il offre une reconstruction des modes de rationalité de l'événement, révélant ainsi toute sa complexité inhérente. Enfin, il propose une redéfinition des notions d'événement, de structure et de conjoncture.

\section{ABSTRACT}

From October 1972 to November 1974, the fate of a large number of Haitian immigrants living in Québec, declared to be illegal aliens by the federal government, emerged as a significant political issue. Using dialogic and synchronic means of analysis, the authors unveil the underlying structural and circumstancial dynamics of the changes affecting Québec society in the first half of the 1970's. Following interpretative approaches inspired by microstoria, this article offers a new reading of the modes of rationality of the event, thus revealing all of its inherent complexity. Finally, the authors propose a reconceptualization of the notions of «event», "structure» and «conjoncture».

1. Les auteurs remercient Andrée Courtemanche, Yves Frenette, Fernand Harvey, Denise Helly, Richard Jones, Kenneth McRoberts, Marcel Martel, Roberto Perin et les deux évaluateurs de la Revue d'histoire de l'Amérique française.

RHAF, vol. 50, n 2, automne 1996 
«Vous croyez que c'est simple, quand on vient d'un pays d'été où tout le monde est noir, de se réveiller dans un pays d'hiver où tout le monde est blanc?»

Dany Laferrière, Chronique de la dérive douce, 97.

Entre le $1^{\text {er }}$ octobre 1967 et le 30 novembre 1972 , le ministère fédéral de la Main-d'œuvre et de l'Immigration offrait aux détenteurs d'un visa de touriste la possibilité de demander le statut de résident permanent sans quitter le territoire canadien. Puis, révoquant ce droit, le ministère canadien oblige les requérants à retourner dans leur pays d'origine pour accomplir cette démarche. Plusieurs ressortissants haïtiens n'ayant pas encore obtenu la citoyenneté tombent ainsi dans l'illégalité et sous le coup d'une ordonnance de déportation.

S'inquiétant du sort réservé aux illégaux, les responsables politiques québécois interviennent auprès des autorités fédérales. Le 12 mars 1973, le ministre québécois de l'Immigration, Jean Bienvenue, demande à son homologue fédéral, Robert Andras, de leur accorder le statut de réfugié politique. Le 10 avril, Andras refuse. Pour Ottawa, il n'y a pas d'exception à faire pour un groupe particulier, et ces migrants auraient des motivations strictement économiques.

Néanmoins, la révocation du règlement fédéral cause des problèmes d'intendance. En juin 1973, Andras estime que de 25000 à 30000 personnes menacées de déportation se présenteront devant la Cour d'appel de l'immigration avant la fin de l'année. Il faut réagir. Le 27 juillet, Ottawa abolit le droit d'appel des visiteurs sous ordonnance de déportation. En août, avec la loi C-197, il lance l' «Opération mon pays», par laquelle les immigrants illégaux peuvent, jusqu'au 15 octobre 1973, régulariser leur situation. Cependant, plusieurs Haïtiens arrivés après le 30 novembre 1972, n'ont pas pu ou su se prévaloir de cette procédure. La Commission d'appel de l'immigration étudie leurs dossiers et, neuf fois sur dix, demande l'exécution immédiate de l'ordonnance. Mille trente-quatre personnes sont touchées par cette mesure.

Au Québec, la situation de ces Haïtiens émeut. Ceux-ci s'intègrent à la communauté francophone. Au nom de principes autant humanitaires que nationalistes, des intervenants au sein de la société civile québécoise, des centrales syndicales à la Conférence des évêques du Canada, se mobilisent en faisant appel à l'opinion publique. En août 1974, sous le leadership du père Paul Dejean et de la Ligue des droits 
de l'homme, ils demandent aux responsables politiques québécois d'intercéder auprès d'Ottawa. À l'automne 1974, ces derniers reprennent contact avec leurs homologues fédéraux. Peine perdue. Le 29 octobre, Bienvenue, pusillanime, cède devant la prépondérance du fédéral en la matière. Le 26 novembre, les parlementaires québécois entérinent cette soumission, malgré un débat orageux.

La Ligue des droits de l'homme et la communauté haïtienne de Montréal réussiront là où le ministère québécois a échoué. En mars 1975, la Ligue présente un dossier accablant sur les jugements de la Cour d'appel de l'immigration et sur les excès de pouvoir des responsables politiques canadiens. Ottawa étouffe l'affaire en réglant discrètement les cas des illégaux un à un, de sorte qu'une infime minorité des Haïtiens menacés d'expulsion le seront effectivement.

Au premier abord, telle une illustration sur une tapisserie, l'intrigue sous-tendant l'événement semble simple. Pourtant, plus l'observateur se rapproche, scrute le motif, change de perspective, plus son regard saisit le miroitement des couleurs, la texture de la pièce, les lacis de la réalité passée, la complexité du cas. En adoptant des principes dialogique et synchronique d'analyse ${ }^{2}$, il est possible de discerner, à travers l'événement des immigrants illégaux haïtiens, affaire fortement documentée ${ }^{3}$, les différentes facettes du changement social, ainsi que ses dynamiques structurelles et conjoncturelles.

En s'inspirant des approches de la microstoria, l'interprétation des indices permet de reconstruire les modes de rationalité, dépositaires de sens, soutenant ces tendances du changement social ${ }^{4}$. L'événement des immigrants illégaux haïtiens, se configurant comme synthèse de l'hétérogène $\mathrm{e}^{5}$, révèle toute la complexité et la chaleur qui se dégagent des dynamiques du temps court. Son importance subjective varie selon la perspective de l'intervenant et de l'analyste, chacun ayant ses préoc-

2. Mikhaïl Bakhtine, Esthétique de la création verbale (Paris, Gallimard, 1984), 158; Edgar Morin, Introduction à la pensée complexe (Paris, ESF, 1990), 98-99.

3. Les Archives nationales du Québec à Montréal conservent le fonds $\mathrm{E} 47 \mathrm{du}$ ministère de l'Immigration du Québec, pour la période 1968-1976. Elles comprennent les documents administratifs provenant de l'interne, des autres ministères et de l'État fédéral. Les auteurs remercient Jean Bienvenue de leur avoir permis de consulter, citer et reproduire les documents pertinents à cette recherche. Les Débats de l'Assemblée nationale du Québec, désormais les $D A N Q$, les quotidiens et les hebdomadaires de la presse écrite québécoise ont été soumis à un dépouillement exhaustif pour la période de novembre 1971 à octobre 1975.

4. Carlo Ginzburg et Carlo Poni, «La micro-histoire», Le Débat, 17 (1981): 133-136; Giovanni Levi, «On Microhistory», dans Peter Burke, dir., New Perspectives on Historical Writing (University Park, PUS Press, 1991), 93-113; Jacques Revel, dir., Jeux d'échelles. La micro-analyse à l'expérience (Paris, Hautes Études/Seuil/Gallimard, 1996), 243 p.

5. Paul Ricœur, Temps et récit (Paris, Seuil, 1985), 1: 102-103. 
cupations propres. Toutefois, en réduisant l'échelle de l'observation, l'historien placé devant l'événement devient plus sensible à la construction du réel et au rôle joué par l'observateur et ses instruments. Désormais, les notions de structure et de conjoncture s'insèrent dans la configuration de l'intrigue, dans ce système complexe et mouvant. Considérons ici les structures comme des dynamiques organisées selon une logique linéaire, ayant une certaine stabilité, encadrant, grâce aux relations déterministes entre leurs éléments, la formulation des objectifs et l'élaboration des stratégies des acteurs. Ainsi, la perspective temporelle pouvant changer, des phénomènes macrosociologiques comme les processus migratoires, des systèmes normatifs stables tels que les lois et les règlements, orientent et structurent l'action. La conjoncture, elle, devient une dynamique non linéaire et aléatoire, donc plus instable, agissant sur les acteurs à l'instar de variables issues de relations déterminables. Aussi, des intrigues se configurant de façon concomitante à celle constituant l'objet de l'analyse et convergeant vers celle-ci, peuvent jouer le rôle d'éléments conjoncturels. Encore là, la durée peut varier, quoique ces dynamiques soient foncièrement instables, voire éphémères, le temps court demeurant souvent la caractéristique première de la conjoncture.

L'étude de la complexité de l'événement des immigrants illégaux haïtiens débute avec les principaux acteurs, les institutions étatiques et les responsables politiques québécois. Ensuite, elle observe le mouvement des structures sur des plans exogènes et endogènes à l'État québécois. Puis elle se penche sur les dynamiques des conjonctures au Québec et au Canada au début des années 1970, qui orientent de façon aléatoire l'ordre du jour des acteurs concernés. Tous ces éléments restent complexes et singuliers à la fois. Cependant, pour se configurer dans le temps, ils s'interpénètrent, échangent, se nouent inextricablement. Ils présentent à l'observateur un spectacle global, une partie fixe du temps prenant un sens à ses yeux.

\section{PRÉSENTATION DES ACTEURS}

Tout au long de l'événement des immigrants illégaux haïtiens, le principal acteur collectif est l'État provincial du Québec, qui possède sui generis les attributs étatiques malgré sa souveraineté partielle. Par sa représentation comme État nation depuis la Révolution tranquille, il joue un rôle de définisseur d'une référence collective, marquant l'identité par l'émission de symboles et de statuts ${ }^{6}$. Il s'implique sur-

6. Gérard Bergeron, Petit traité de l'État (Paris, Presses universitaires de France, 1990), 14-15; Pierre Bourdieu, «Esprit d'État. Genèse et structure du champ bureaucratique», Raisons 
tout par le biais de deux institutions, lieux de définition de valeurs communes $^{7}$, modelant les politiques et modelées par l'histoire. D'abord, l'Assemblée nationale, «forum de la Nation ${ }^{8}$ », reflète dans ses débats les différents enjeux de l'événement. Ensuite, le ministère de l'Immigration, infrastructure administrative relevant des fonctions de l'exécutif, intervient selon quatre relations dynamiques, comme autant de strates de complexité. Au centre, il y a l'organisation administrative du ministère même. Puis, se dirigeant vers la périphérie, se situent les dynamiques intra et interétatiques. Enfin, les relations avec la société civile constituent la dernière strate. Dispersés dans cet entrelacs complexe, les acteurs étatiques mettent en place un système normatif assurant la régulation des rapports sociaux.

Ces dynamiques institutionnelles reflètent également les tendances, ruptures et continuités présentes au sein de la culture politique québécoise, tels le processus de modernisation de l'État québécois ${ }^{9}$ ou les transformations des rapports entre l'appareil étatique et le citoyen. La culture politique passe d'une dialectique plus informelle du népotisme, où la redistribution des ressources est fonction de la loyauté partisane, à un système marchand, où des rapports formalisés se nouent entre un État producteur et des usagers, clients ou bénéficiaires ${ }^{10}$. Recherchant l'adhésion du citoyen, l'État produit aussi une économie générale du symbole, qui prend de nouveaux sens. Délaissant une représentation organique de la communauté politique, axée autour des référents de l'ethnie et de la religion, les acteurs étatiques adoptent progressivement une représentation contractuelle où le pluralisme, la démocratie, le droit, l'économie deviennent des phares ${ }^{11}$.

pratiques (Paris, Seuil, 1994), 107; Fernand Dumont, Genèse de la société québécoise (Montréal, Boréal, 1993), 342-348; Lucien Sfez, La politique symbolique (Paris, Presses universitaires de France, 1993) (1978), 273-486.

7. Charles Taylor, «Les institutions dans la vie nationale», dans Vincent Lemieux, dir., Les institutions québécoises, leur rôle, leur avenir (Sainte-Foy, Presses de l'Université Laval, 1990), 52.

8. A. Paul Pross, Group Politics and Public Policy (Toronto, Oxford University Press, 1992), 252.

9. Kenneth McRoberts, Quebec: Social Change and Political Crisis (Toronto, McClelland and Stewart, 1988), 131-139.

10. Ralph Heinzmann, «The Political Culture of Quebec, 1840-1960», Canadian Journal of Political Science, 16,1 (1983): 3-59; Jacques T. Godbout, La participation contre la démocratie (Montréal, Albert Saint-Martin, 1983), 190 p., et La démocratie des usagers (Montréal, Boréal, 1987), $192 \mathrm{p}$.

11. Martin Pâquet, Le Fleuve et la Cité. Représentations de l'immigration et esquisses d'une action de l'État québécois, 1945-1968, thèse de doctorat (histoire), Université Laval, 1995, 384-391. 
Se limiter aux acteurs collectifs et institutionnels serait opérer une simplification abusive. Élus et cognoscentis ${ }^{12}$, les responsables politiques au sein du système de gouverne exercent des pouvoirs généraux par leur habilitation à prendre des décisions dans la régulation des situations à problèmes. Leurs interrelations personnelles influent sur les différentes dynamiques en cause, déterminant la fréquence et la qualité de l'information échangée. Les relations cordiales entre les responsables fédéraux et provinciaux influencent sûrement le déroulement de l'événement des immigrants illégaux.

\section{L'ÉVÉNEMENT RÉVÉLATEUR DES TRANSFORMATIONS STRUCTURELLES}

Présentes dans l'événement des ressortissants haïtiens, révélées par celui-ci, diverses dynamiques structurelles orientent les objectifs et les stratégies des acteurs politiques, collectifs et individuels. Animées par différentes logiques, elles dialoguent entre elles, se modifient, s'intègrent à des degrés divers, à des temporalités variables. Ces aspects linéaires des changements sociaux constituent une première dimension de complexité à l'intérieur de cette synthèse de l'hétérogène. Ils renvoient à la compréhension du mouvement migratoire, puis aux considérations domestiques de l'État provincial.

\section{L'émigration haïtienne}

En 1957, François Duvalier prend le pouvoir. S'affûtent alors les serres d'un «État-prédateur», selon l'expression de Mats Lundahl. D'une émigration temporaire et rurale vers les plantations de canne à sucre de Cuba et de la République dominicaine, le mouvement prend un caractère plus systématique et permanent. Une diaspora se constitue sous la pression des mesures de rétorsion contre les opposants politiques ainsi que la double désintégration de la situation économique et de la société civile. Après 1964, au moment où «Papa Doc» se déclare président à vie, le mouvement d'émigration s'étend des membres de l'élite urbaine aux ouvriers qualifiés. Dès que JeanClaude Duvalier remplace son père en 1971, l' «État prédateur» haïtien bloque toute possibilité de mobilité sociale. Confrontés à une misère extrême, les ouvriers non qualifiés et les paysans prennent massivement le chemin de l'exil. En 1982, un million d'Haïtiens, soit une personne sur six, vivent à l'étranger, surtout aux États-Unis et en

12. Nimbés du capital symbolique de l'expertise et de la compétence reconnues, les détenteurs de la connaissance ou cognoscentis appartiennent à la fonction publique ou conseillent de l'extérieur le système de gouverne. Ibid., 119-120. 
République dominicaine. Troisième foyer en importance de la diaspora, le Canada accueille 40000 ressortissants ${ }^{13}$.

Dès 1967, informés par des missionnaires canadiens-français, plusieurs émigrants haïtiens optent pour le Québec. Souvent détenteurs d'un visa de touriste, ils bénéficient de la libéralisation offerte par les règlements fédéraux. Cette émigration suit quantitativement et qualitativement le même modèle que le mouvement d'ensemble. En chiffres absolus, leur nombre augmente sans cesse jusqu'en 1974 (voir tableau 1). S'établissant surtout dans la région montréalaise, les ressortissants haïtiens occupent le premier rang des immigrants reçus au Québec dès 19744.

Tableau 1

Immigrants nés à Haïti et admis au Québec 1968-1975

$\begin{array}{cccccccc}1968 & 1969 & 1970 & 1971 & 1972 & 1973 & 1974 & 1975 \\ 568 & 658 & 908 & 1060 & 1009 & 2266 & 4856 & 3422\end{array}$

Plusieurs immigrants d'implantation récente espèrent retourner dans la «perle des Antilles» lorsque les conditions seront plus propices. Entre-temps, un réseau institutionnel se forme progressivement autour de la famille, de groupements politiques et d'associations catholiques, tissant des liens avec la société d'accueil. La menace de déportation survient au cours de ce processus d'intégration, cristallisant ainsi l'identité de la jeune communauté haïtienne-québécoise ${ }^{16}$.

\section{Les considérations domestiques}

1 - Un État à la recherche d'un contrôle démographique

Après la Seconde Guerre mondiale, les individus conçoivent l'État nation comme un cadre d'accueil avec lequel ils nouent des pactes

13. Mats Lundahl, «Underdevelopment in Haiti: Some Recent Contributions», Politics or Markets? Essays on Haitian Underdevelopment (Londres, Routledge, 1992), 46-61; The Haitian Economy. Man, Land and Markets (Londres, Croom Helm, 1983), 164-167; Laënnec Hurbon, Comprendre Haïti. Essais sur l'État, la nation, la culture (Paris, Karthala, 1987), 28-43.

14. André Jacques, «La longue marche des Haïtiens», dans Joseph-Ph. Antonio, dir., Haïti, briser les chaînes (Lausanne, Favre, 1974), 73; Paul Dejean, Les Haïtiens au Québec (Sillery, Presses de l'Université du Québec, 1976), 39-40 et 78-80; Simon Langlois et al., La société québécoise en tendances, 1960-1990 (Québec, Institut québécois de recherche sur la culture, 1990), 588.

15. Dejean, op. cit., 18.

16. Ibid., 143-170. 
temporaires d'intérêt mutuel, résiliables par leur départ. Dans un monde où, en principe, chacun a droit au bonheur et où les frontières sont poreuses, les individus et les entreprises se croient dorénavant libres de rechercher des lieux propices à leur épanouissement. Ces croyances questionnent le primat de la souveraineté étatique. Dès lors, l'État de droit se sent paradoxalement tiraillé entre le maintien d'une permanence et le respect de la règle, soit sa justification et son adhésion premières ${ }^{17}$. Aussi cherche-t-il à contrôler son territoire.

L'affaire des ressortissants haïtiens soulève concrètement ce dilemme. «La souveraineté exclusive, ce vieux dogme», note le chef de l'opposition Jacques-Yvan Morin, s'effrite «devant une certaine idée de la liberté» et «de l'homme». Les autorités fédérales ne partagent pas son avis. Même si Ottawa assure à ses citoyens et aux aubains des droits et des recours, l'État canadien veut contrôler, en révoquant son règlement, la circulation des individus sur son territoire. En octobre 1974, Alan Gotlieb, sous-ministre canadien, use de cet argument pour refuser toute concession. Selon lui, il n'y a «que deux façons de céder dans ce cas, à savoir une amnistie générale» ou l'annulation du règlement de novembre 1972, qui provoqueraient «une invasion d'immigrants illégaux à l'échelle nationale». La seule solution s'avère le renvoi des illégaux, «à l'exception des réfugiés authentiques» reconnus par la législation. Partageant ces vues, Bienvenue adhère sans réticences au strict respect des frontières ${ }^{18}$.

L'État tente également d'exercer son pouvoir sur les populations. En plus d'établir des mécanismes de régulation et de normalisation, il lui faut créer des conditions assurant leur maintien et leur épanouissement. Entre 1972 et 1974, le Québec accuse une dénatalité importante, dont les responsables politiques perçoivent les conséquences sur le développement économique et le poids politique au sein de la Confédération ${ }^{19}$. Il existe une planche de salut, l'immigration internationale, qui représente $24,3 \%$ de l'accroissement de la population nette québé-

17. Philippe Moreau Defarges, Relations internationales, 2: Questions mondiales (Paris, Seuil, 1993), 70-71.

18. Morin, $D A N Q, 15,77$ (7 novembre 1974), 2677. Gotlieb, E 47, art. 3, 1.11. R. L. Wales, Mémorandum à tous les bureaux étrangers M \& I (Ottawa, 27 novembre 1974), 1-2. Bienvenue, $D A N Q, 15,85$ (26 novembre 1974), 2986.

19.. E 47, art. 3, 1.11.1, Québec, Ministère de l'Immigration, La position du gouvernement du Québec à la suite de la publication du livre vert fédéral sur la politique canadienne d'immigration (Québec, s.é., avril 1975), 5-6 et 8-14; art. 4, 1.18, Jean Bienvenue, Les politiques actuelles du ministère de l'Immigration du Québec (26 mars 1974), 2; art. 1, 1.2.3.1, Jean Bienvenue, Objet: Livre blanc sur les ressources humaines (Québec, août 1974), 5; Québec, Ministère de l'Immigration, Pour une politique de population (Québec, s.é., septembre 1975), 170 p.; Denise Helly, L'immigration, pour quoi faire? (Québec, Institut québécois de recherche sur la culture, 1992), 43. 
coise en $1971^{20}$. Pour l'État québécois, la «politique d'immigration est une des composantes d'une politique démographique» garantissant «l'équilibre national, tant au plan régional que linguistique ${ }^{21} »$. Puisque les parents québécois auraient «froidement décidé de ne plus avoir d'enfants», Bienvenue prédit, pontifiant: «En vérité, je vous le dis, l'immigration, c'est l'avenir du Québec ${ }^{22}$.»

Cette option ne va pas sans problèmes. D'abord, les migrants sont souvent de passage, la force d'attraction du Québec jouant peu. Leur nombre absolu tend à diminuer au début des années 1970 (voir tableau 2). En s'installant massivement dans la région montréalaise, les nouveaux arrivants contribuent au déséquilibre spatial. Ils adoptent l'anglais comme langue de communication. Enfin, ils proviennent davantage de l'Asie et des Amériques. Au lieu d'expliquer cette situation par la transformation des mouvements migratoires internationaux, le ministère y voit plutôt les effets des réglementations fédérales touchant l'universalité et le parrainage ${ }^{23}$.

\section{Tableau 2}

Immigrants au Québec, 1969-1975

$\begin{array}{ccccccc}1969 & 1970 & 1971 & 1972 & 1973 & 1974 & 1975 \\ 26789 & 22383 & 17904 & 18815 & 32452 & 31670 & 26629\end{array}$

Un des objectifs du ministre québécois de l'Immigration est de «maîtriser de plus en plus le flux migratoire», en tenant compte du partage des compétences constitutionnelles et de la libre circulation des personnes à l'intérieur du Canada. Bien que les immigrants haïtiens soient francophones, Bienvenue ne peut se «satisfaire d'une immigration qui ne correspond pas adéquatement aux véritables besoins du Québec». En effet, cette immigration serait «trop mécanique [sic]», car elle éviterait le processus normatif de sélection de l'Etat

20. E 47, art. 27, Jean Bienvenue, Politique linguistique et immigration (Québec, s.é., février 1974), 4.

21. E 47, art. 18, Gérard-D. Lévesque, Déclaration sur les consultations intergouvernementales relatives à la démographie et à des questions connexes (Saint-John's, 21-22 août 1975), 3-4.

22. Le Journal de Montréal (11 avril 1974), 20.

23. E 47, art. 27, Bienvenue, Politique linguistique..., 2-4; art. 3, 1.11.1, Québec, Ministère de l'Immigration, La position..., 22-23, 25 et 28; art. 17, 1.1b, s.a., Le Québec et l'immigration (Québec, juillet 1974), 6; Québec, Ministère de l'Immigration, Rapport annuel 1972-1973 (Québec, Éditeur officiel, 1973), 12 et 30; Québec, Ministère de l'Immigration, Rapport annuel 1973-1974 (Québec, Éditeur officiel, 1974), 14. 
québécois ${ }^{24}$. Aussi, il lui faut privilégier la sélection individuelle, mieux adaptée aux problèmes démographiques, pour réduire les effets du système de parrainage et la répétition de cas similaires aux ressortissants haïtiens ${ }^{25}$.

\section{2 - Un État rationnel à la recherche du progrès économique}

En élaborant un cadre normatif d'inclusion et d'exclusion de l'Étranger, les responsables politiques se réfèrent à leur gestion de l'économie. Une main-d'œuvre qualifiée et le potentiel d'investissements forment autant de normes valorisées jaugeant l'apport de l'immigrant au développement économique. Au ministère de l'Immigration, élus et cognoscentis se représentent l'immigrant idéal sous les traits de l'homo æeconomicus, doté de compétences professionnelles et détenteur d'un capital financier ${ }^{26}$. Malgré la conciliation malaisée des besoins de la démographie et de l'emploi, toute action étatique entend se coller de près à l'évolution interne du marché du travail, en croissance de 1960 à 1973 . Les immigrants constituent une forte proportion de l'augmentation annuelle de la population active, soit $28 \%$ de l'ensemble ${ }^{27}$. Ils doivent donc s'insérer dans les stratégies globales de l'État québécois en matière de main-d'œuvre ${ }^{28}$.

Ici, le ministère québécois de l'Immigration remplit un rôle moins important que celui de son homologue fédéral. Il ne détient pas 'exclusivité du dossier de la main-d'œuvre et l'État provincial doit d'abord combler les besoins d'emploi des travailleurs locaux. Pourtant, le ministère québécois n'est pas sans utilité. Il informe les immigrants des conditions de l'emploi, et s'implique aussi dans l'identification et la planification des besoins conjoncturels du marché du travail québécois. En 1974 et 1975, un des sujets majeurs de discussion entre les fonctionnaires fédéraux et provinciaux concerne la sélection économique des immigrants. Cette sélection, même imparfaite, se fonde sur la demande interne de main-d'œuvre qu'il importe de réguler le mieux possible. Dès sa création, le ministère de l'Immigration élabore un protocole à ce sujet. Même si l'intégration des immigrants doit être facilitée, les fonctionnaires doivent toujours

24. E 47, art. 26, Jean Bienvenue, Notes pour une allocation [sic] du ministre (Québec, 6 mai 1975), 13.

25. E 47, art. 2, 1.7.1, Jean Bienvenue, Conférence de presse prononcée par Me Jean Bienvenue (Québec, 23 mai 1975), 8-12.

26. E 47, art. 27, Bienvenue, Politique linguistique..., 5.

27. E 47, art. 2, 1.7.1, Bienvenue, Conférence de presse..., 8-12.

28. E 47, art. 27, Bienvenue, Politique linguistique..., 5; art. 3, 1.11.1, Québec, Ministère de l'Immigration, La position..., 2; art. 18, Lévesque, Déclaration..., 3. 
vérifier si un citoyen canadien ne peut combler le poste avant un résident temporaire non immigrant. Ensuite, le ministère peut privilégier, dans sa sélection, des jeunes francophones ou «francophonisables», instruits, dynamiques et créateurs d'emplois. Source par excellence de ces immigrants, la France pourrait les orienter vers son partenaire privilégié en Amérique du nord ${ }^{29}$. Ce qui n'exclut pas les anglophones, bien au contraire, puisque les professionnels et techniciens britanniques, «hautement spécialisés», «sont particulièrement intéressants pour le Québec ${ }^{30}$ ».

Second visage de l'homo ceconomicus, l'investisseur apparaît comme un candidat de choix, pour les élus et les cognoscentis québécois $^{31}$. En 1972, le Ministère se dote d'un service des immigrants entrepreneurs, qui devient le pourvoyeur idéal de ces citoyens «de qualité», «éléments essentiels du développement économique du Québec». Aux yeux des responsables politiques, ce service s'avère d'une rentabilité certaine ${ }^{32}$. Pour l'année financière 1974-1975, 239 immigrants investisseurs, originaires de 44 pays, s'établissent au Québec. Cent trente-trois émigrent de France. Un seul provient d'Haïti ${ }^{33}$. Comme il faut recruter et sélectionner des immigrants «dans des pays dont l'apport au développement économique et social sera réel ${ }^{34}{ } \gg$, les ressortissants haïtiens sont défavorisés. Ils ne correspondent pas au profil valorisé de l'immigrant latino-américain, provenant des «classes moyennes et supérieures», doté de capitaux ${ }^{35}$. Ce sont le plus souvent des «citoyens plus modestes», «à l'emploi d'usines et d'entreprises, à Montréal et ailleurs au Québec», qui sont menacés par les déportations ${ }^{36}$.

Heureusement pour les immigrants illégaux, la poursuite rationnelle du progrès économique et la représentation instrumentale de

29. E 47, art. 2, 1.6.1, René Didier à Jean Bienvenue, Compte rendu du voyage en Europe du 24 au 27 septembre (Montréal, 7 octobre 1974), 5 p.

30. E 47, art. 7, 2.2, René Gagnon à Jean Bienvenue, Points abordés avec MM. Denis Vaugeois et Jean Primeau (Québec, 31 janvier 1973), 3-4.

31. E 47, art. 26, Bienvenue, Notes..., 8-9.

32. E 47, art. 4, 1.18, Jean Bienvenue, Le ministère de l'Immigration du Québec et les immigrants investisseurs (Montréal, 10 avril 1974), 6-17.

33. E 47, art. 26, Bienvenue, Notes..., 17-18; art. 4, 1.18, Bienvenue, Le ministère..., 19 p.; art. 17, 1.1, Jean Bienvenue, "Québec-industriel» (Québec, 1972), 6-7; art. 29, s.a., Compte-rendu de la mission des membres de l'Assemblée nationale du Québec sur les problèmes de l'immigration (Montréal, 22 octobre 1975), 3; Québec, Ministère de l'Immigration, Rapport annuel 1974-1975 (Québec, Éditeur officiel, 1975), 65.

34. E 47, art. 3, 1.11.1, Québec, Ministère de l'Immigration, La position..., 46.

35. E 47, art. 25, s.a., Notes sur le potentiel de l'Amérique du sud pour l'immigration au Québec (Québec, 5 mars 1972), 2.

36. Cité par Jacques-Yvan Morin, DANQ, 15,85 (26 novembre 1974), 2978-2979. 
l'homo æcconomicus ne sont ni dogmatiques ni limitées au court terme. Bienvenue lui-même montre une certaine souplesse en subordonnant l'impératif de la croissance économique à tout prix à celui de la «qualité de vie» de la société québécoise. À cette fin, il propose une réforme en profondeur de la politique de main-d'œuvre. "Tout en assurant aux Québécois un bon niveau de vie», elle favoriserait «leur épanouissement dans les régions qu'ils habitent, de façon à préserver et à rendre ainsi significatives et culturellement fécondes les solidarités naturelles de base ${ }^{37} \gg$. Cette réforme ferait une place à des immigrants pauvres, peu instruits, tels que nombre d'Haïtiens quittant leur pays en ruine.

\section{3 - Un État producteur de normes et de symboles}

Au tournant des années 1960, la francophonie constitue une cible de choix des stratégies étatiques. Déjà en 1970, l'Agence de coopération culturelle et technique reconnaît le Québec comme "gouvernement participant». Après un détour vers les préoccupations économiques au cours du premier mandat du gouvernement Bourassa ${ }^{38}$, l'élection d'octobre 1973 marque le retour de l'intérêt québécois vers la francophonie, par une présence accrue dans les régions où se situent les bassins d'une immigration d'expression française ${ }^{39}$. De plus, l'Amérique latine devient une cible de politique extérieure dès $1973^{40}$. «Compte tenu des affinités naturelles des immigrants sud-américains», l'État québécois espère même «recevoir de 40 à $50 \%$ de tous ceux qui s'établissent au Canada». En mars 1974, Jean Bienvenue annonce la nomination éventuelle d'agents d'immigration en Amérique latine ${ }^{41}$. De son côté, Gérard-D. Lévesque, ministre des Affaires intergouvernementales, fixe les objectifs généraux de l'État provincial, en voulant assurer une plus grande présence québécoise, en particulier dans le secteur de la coopération. Pour des raisons de langue et de culture, Lévesque joint Haïti à sa liste des pays latino-américains. Ce pays accueille d'ailleurs le premier bureau d'immigration, établi à Port-auPrince à la fin de l'année $1974^{42}$.

37. E 47, art. 1, 1.2.3.1, Bienvenue, Objet..., 4-5.

38. Ivan Bernier, «De l'économie mondiale à la francophonie: les cibles générales et institutionnelles», dans Louis Balthazar et al., Trente ans de politique extérieure du Québec, 1960-1990 (Sillery, CQRI/Septentrion, 1993), 325-326.

39. E 47, art. 4, 1.18, Bienvenue, Les politiques actuelles..., 11; La Presse, 20 novembre 1974, 3 .

40. E 47, art. 7, 2.2, René Gagnon à Jean Bienvenue, Points abordés..., 3; Gordon Mace, «Les relations du Québec avec l'Amérique latine», dans Balthazar et al., op. cit., 225 et 228.

41. E 47, art. 4, 1.18, Bienvenue, Les politiques actuelles..., 10.

42 E 47, art. 7, 2.2, s.a., Agents d'information à l'étranger (Entente Cloutier-Lang) (Montréal, s.é., 11 avril 1973), 2; art. 3, 1.11, Alan E. Gotlieb et René Didier, Problèmes géné- 
L'affaire des immigrants illégaux a ainsi un impact direct sur la présence du Québec à l'étranger. À défaut de régler le cas des illégaux menacés d'expulsion, les sous-ministres canadien et québécois, Alan Gotlieb et René Didier, s'entendent sur un moyen d'éviter que pareille situation ne se reproduise, avec l'ouverture d'un poste d'agent d'immigration du Québec à Port-au-Prince ${ }^{43}$. Néanmoins, l'intérêt de l'État québécois à l'endroit d'Haïti demeure circonspect, en raison de la nature dictatoriale du régime Duvalier ${ }^{44}$, des incidences politiques internes de l'affaire des ressortissants et des budgets limités des ministères concernés. La diplomatie québécoise des années 1970 montre sa réserve en n'instaurant que des programmes ponctuels de coopération. Cependant, ces programmes lui valent un certain crédit politique, d' où leur rentabilité symbolique ${ }^{45}$.

La gestion de l'immigration renvoie aussi aux fonctions symbolique et normative de l'État provincial. Après la motion Loubier et la création d'un service d'Immigration au sein du ministère des Affaires culturelles en 1965, les responsables politiques québécois mettent sur pied une bureaucratie chargée de réguler l'admission des nouveaux venus au Québec. En novembre 1968, la création du ministère de l'Immigration, geste hautement symbolique, établit un cadre administratif. Dès lors, la stratégie québécoise consiste à obtenir d'Ottawa la reconnaissance des compétences provinciales.

Le 18 mai 1971, une entente est conclue entre les ministres canadien et québécois Otto Lang et François Cloutier. Elle permet à l'État provincial de nommer des agents d'orientation qui œuvreront à l'intérieur de certaines ambassades canadiennes. Ces agents ne s'occuperont pas de recrutement mais seulement d'information. Les fonctionnaires canadiens des services des ambassades conserveront le privilège exclusif d'émettre des visas ${ }^{46}$.

L'entente Cloutier-Lang n'a pas vraiment une grande portée. En décembre 1971 , le ministère québécois procède à la nomination de fonctionnaires dans les bureaux de trois ambassades canadiennes seulement. À la suite des critiques virulentes sur le rôle de second violon de l'État québécois, la commission d'enquête sur la situation de la

raux-1 (Ottawa, 10 octobre 1974), 3 et 6; Le Devoir, 20 novembre 1974, 1-2; André Patry, Le Québec dans le monde (Ottawa, Leméac, 1980), 136.

43. E 47, art. 4, 1.13.1, René Didier à Jean Bienvenue, Montréal, 28 octobre 1974, 2.

44. DANQ, 15,90, 4 décembre 1974, 3161.

45. Claude Morin, L'art de l'impossible (Montréal, Boréal, 1987), 300-302.

46. Québec, Ministère de l'Immigration, Rapport annuel 1971-1972 (Québec, Éditeur officiel, 1972), 7-8; ANQQ, E 5, Fonds du Conseil exécutif, art. 8; François Cloutier et Otto Lang, Entente générale/General Agreement (Québec-Ottawa, s.é., 18 mai 1971), 1-7. 
langue française et sur les droits linguistiques au Québec (commission Gendron) propose la révision des termes de l'entente ${ }^{47}$. Cette contestation incite le nouveau ministre Bienvenue à entamer une renégociation avec le ministère fédéral. L'éventuelle entente devrait donner des moyens à l'action québécoise, orientée selon le triptyque suivant: «une information adéquate des immigrants, un pouvoir réel de sélection et de recrutement» ainsi qu' «une intégration harmonieuse à la société québécoise ${ }^{48} \gg$. Présenté à l'Assemblée nationale en juillet 1974, le projet de loi 46 affirme la volonté du Québec d'agir en ce sens. Plus encore, il prévoit la restructuration du ministère de l'Immigration, qui devrait devenir celui «de la Population» ou «des Ressources humaines ${ }^{49}$ ».

Ottawa se sent disposé à coopérer, mais à ses conditions. En octobre 1974, Gotlieb rappelle la compétence exclusive de l'État fédéral en matière d'admission au pays, ouvrant néanmoins la porte à l'accroissement des activités de la Province au «stade préalable à l'admission, tels la publicité, l'encouragement et la présélection ${ }^{50}{ }$. Cette ouverture attend sa contrepartie. La décision finale de Bienvenue dans le cas des ressortissants haïtiens reflète sa volonté de compromis. «En conséquence», reconnaît le ministre le 26 novembre 1974, «le Québec, comme toute autre province, ne peut prendre des mesures pour empêcher» toute expulsion. «C'est de juridiction [sic] fédérale, et toujours fédérale ${ }^{51} . »$

Dans ces conditions, Andras, le ministre canadien, accepte le principe de la participation des agents québécois aux activités relatives aux renseignements, à la sélection et au recrutement des immigrants ${ }^{52}$. Les négociations reprennent et, le 17 octobre 1975, Andras et Bienvenue signent une entente qui consacre les nouvelles compétences québécoises. Cette entente constitue une étape importante dans la constitution d'un État québécois producteur de normes sociales d'inclusion.

47. Le Devoir, 19 mai 1971, 4; 20 mai 1971, 3; 21 mai 1971, 4; 29 mai 1971, 6. Québec, Commission d'enquête sur la situation de la langue française et sur les droits linguistiques au Québec, Rapport, livre III, Les groupes ethniques et l'épanouissement du français au Québec (Québec, Éditeur officiel, décembre 1972), 132.

48. E 47, art. 4, 1.18, Bienvenue, Les politiques actuelles..., 3 et 8-11; Le Jour, 23 mai 1974, 3; Le Devoir, 28 mars 1974, 5.

49. Le Devoir, $1^{\text {er }}$ août 1974,1 et 6.

50. E 47, art. 3, 1.11; R. L. Wales, Mémorandum..., 1-2.

51. DANQ, 15,85 (26 novembre 1974), 2981.

52. E 47, art. 3, 1.11, Robert Andras à Jean Bienvenue, Ottawa, 12 février 1975, 1. 


\section{L'ÉVÉNEMENT RÉVÉLATEUR}

\section{DES TRANSFORMATIONS CONJONCTURELLES}

Dévoilant les mutations structurelles, l'événement des immigrants illégaux haïtiens révèle également les changements sociaux non linéaires soulevés par les débats conjoncturels. Qu'ils touchent à l'enjeu constitutionnel, au multiculturalisme, à l'altérité, à la question linguistique ou à la dimension des droits de la personne, ces débats influent de façon aléatoire sur les objectifs et les stratégies des responsables politiques québécois. Ces débats polémiques ont une logique propre et des relations dialogiques. Partie prenante de cette conjoncture, de cette autre dimension de complexité, l'événement se trouve à la conjonction de ces débats, qui le traversent, le dirigent, voire le bousculent.

\section{La stratégie de la souveraineté culturelle}

\section{1 - Les enjeux constitutionnels}

En 1970, les libéraux de Robert Bourassa adhèrent toujours à la conception de la dualité canadienne, où le Québec, berceau de la nation et de la culture canadiennes-françaises, se voit investi de responsabilités particulières. Suivant celles-ci, il importerait à l'État québécois de réformer le cadre confédératif, de manière à y inclure les aspirations nationales du Québec à l'intérieur d'une constitution renouvelée. Or, les priorités d'Ottawa sont fort différentes. L'État fédéral poursuit sa centralisation des pouvoirs amorcée depuis la Seconde Guerre mondiale. Avec l'arrivée au pouvoir de Pierre Elliott Trudeau, une nouvelle stratégie s'élabore pour contrer le nationalisme québécois, incompatible avec les institutions politiques canadiennes ${ }^{53}$. Les deux visions s'affrontent à la conférence constitutionnelle de Victoria de juin 1971. Après l'échec de la conférence, chaque partie doit revoir ses modalités d'action. Robert Bourassa présente alors la nouvelle stratégie québécoise, dite de la souveraineté culturelle. Il n'est plus question de discuter d'amendements constitutionnels. Désormais, le Québec tente d'atteindre les objectifs fixés par des lois provinciales et par la négociation d'ententes administratives avec l'État canadien.

L'immigration constitue l'une des pierres angulaires de la souveraineté culturelle. De 1971 à 1976, le ministre Bienvenue et les cognoscentis se réclament de la dualité culturelle entre les deux peuples

53. Kenneth McRoberts, English Canada and Quebec. Avoiding the Issue (North York, Robarts Center for Canadian Studies, 1991), 14. 
fondateurs ${ }^{54}$. Comme «la constitution ne fixe en aucun cas de contenu et laisse libre la détermination de ce contenu ${ }^{55}$ », «toute politique canadienne d'immigration» devrait «requérir une participation active des provinces» pour la sélection et le recrutement. L'intégration devrait relever de «la responsabilité prioritaire des provinces ${ }^{56}{ }$. La souveraineté culturelle fournit une légitimité aux revendications du ministère de l'Immigration. Dans ce cadre, affirme Bienvenue en mars 1974, «le Québec entend récupérer d'autres morceaux de juridiction [sic]», notamment le choix et l'orientation des nouveaux citoyens ${ }^{57}$. Le remplacement de l'entente Cloutier-Lang s'inscrit également dans cette stratégie, tout comme le projet de loi 46 déposé en juillet $1974^{58}$.

L'événement des ressortissants haïtiens se déroule dans un contexte de tractations entre Québec et Ottawa. Ces négociations se font en deux temps. De novembre 1972 à juin 1974, les négociateurs québécois ont l'avantage puisque le gouvernement Trudeau est minoritaire à la Chambre des communes. Ottawa ne prend aucune initiative constitutionnelle, tout comme il n'entreprend pas de réorganisation administrative majeure. Aussi, Bienvenue peut faire des pressions sur son homologue fédéral pour régler le problème des immigrants illégaux dès mars 1973. L'«Opération mon pays» et l'étude des dossiers des ressortissants haïtiens accordent par la suite un répit aux responsables canadiens. Au début de 1974, tout en reconnaissant la prépondérance fédérale dans le dossier des ressortissants haïtiens, les cognoscentis québécois vitupèrent contre les décisions d'Ottawa et sa gestion de l'immigration. Aussi, le 15 mai 1974, Québec exige une nouvelle entente portant sur «l'information, le recrutement et la sélection des ressortissants étrangers destinés à la résidence permanente ou temporaire au Québec ${ }^{59}{ }^{\prime}$. Enfin, les responsables politiques québécois réclament le droit «de s'opposer à des candidats qui ne correspondent pas à ses besoins». Sinon, affirme Bienvenue le 26 mars 1974 à l'Assemblée nationale, l'État provincial devrait «légiférer sur un éventuel permis de travail». Le ministre insiste en exigeant, dans un mémoire à Andras, que les agents d'orientation du Québec «puissent

54. E 47, art. 4, 1.18, Bienvenue, Les politiques actuelles..., 1-2.

55. E 47, art. 3, 1.10.5, René Didier, Le rappatriement [sic] des pouvoirs du Québec en matière d'immigration (Québec, 23 septembre 1975), 6.

56. E 47, art. 18, Lévesque, Déclaration..., 4-5.

57. Le Devoir, 14 mars 1974, 11.

58. E 47 , art. 26, Bienvenue, Notes..., 5.

59. E 47, art. 3, 1.10.2, Arthur Tremblay à René Gauthier, Québec, 6 mars 1974, 17-18; art. 3, 1.10.5, Didier, Le rappatriement [sic]..., 1-2. 
obtenir le rejet de certaines candidatures ${ }^{60} \gg$. Robert Bourassa va dans ce sens le 26 mai $1974^{61}$.

Le vent tourne avec la campagne électorale fédérale qui bat son plein en juin 1974. Sentant la victoire proche, les libéraux fédéraux manifestent une opposition ferme aux rodomontades de Bienvenue. Robert Andras réplique. «La proposition de veto est inacceptable», tonne-t-il, car «il n'existe pas, constitutionnellement, de moyen par lequel nous pourrions confier un pouvoir de veto à une province en matière d'immigration, celle-ci étant clairement de juridiction [sic] fédérale ${ }^{62} \gg$. Bienvenue se défend ensuite de telles intentions, admettant que «le Québec ne peut obtenir de droit de veto sur la libre circulation des immigrants entrés au Canada». Au contraire, plaide le ministre, c'est «un droit de regard», jugé plus «positif», que la Province veut acquérir ${ }^{63}$.

En maintenant sa position, Ottawa désire apaiser les tensions en reconnaissant, dans le Livre vert du ministre Andras à l'automne 1974, la légitimité des objectifs spécifiques du Québec. Bienvenue montre parallèlement une souplesse surprenante dans le dossier des ressortissants haïtiens. En effet, concède-t-il, «les décisions concernant l'émission des visas d'immigration, l'expulsion d'une personne en contravention avec la loi fédérale de l'immigration, la poursuite des procédures en appel et l'application des décisions finales sont de la compétence exclusive et de la pleine juridiction [sic] du gouvernement $\mathrm{d}^{\prime} O$ ttawa $^{64}{ }^{7}$. À ceux qui s'étonnent de cette reconnaissance sans équivoque, le ministre en appelle au réalisme, car il ne faudrait pas «aller jusqu'à l'humiliation totale, qui ne serait pas bonne pour le Québec dans le contexte actuel ${ }^{65} \gg$. Au cours du débat parlementaire, Bienvenue n'hésite pas à évoquer l'aliénation du territoire québécois, permettant aux ressortissants haïtiens de satisfaire à l'exigence de la loi canadienne $^{66}$. L'opposition réagit vivement à cette suggestion «partitionniste». Selon Jacques-Yvan Morin, les compétences «exclusives [du fédéral] ne lui sont pas dévolues par la constitution». «Si Québec posait les mêmes gestes» qu'Ottawa, l'État québécois «pourrait demain exercer ses compétences» en cette matière ${ }^{67}$.

60 La Presse, $1^{\text {er }}$ novembre 1975, A-5.

61. Ibid.; Le Devoir, 15 juin 1974, 3.

62. Le Devoir, 14 juin 1974, 2.

63. Le Devoir, 15 juin 1974, 3 .

64. DANQ 15,71 (29 octobre 1974), 2398; E 47, art. 3, 1.10.5, Didier, Le rappatriement [sic]..., 7-8.

65. DANQ, 15,78 (8 novembre 1974), 2707.

66. Le Devoir, 19 novembre 1974, 3.

67. $D A N Q, 15,78$ (8 novembre 1974), 2707-2708. 


\section{2 - La contestation du multiculturalisme}

La principale charge contre la souveraineté culturelle provient d'Ottawa. Pour permettre une meilleure identification du citoyen à l'endroit de l'État canadien, celui-ci transforme son ordre symbolique, en particulier dans le caractère normatif des institutions fédérales. Dès les années 1960, avec la Commission Laurendeau-Dunton et la Loi sur les langues officielles, ce nouvel ordre symbolique cherche à intégrer les francophones vivant au Canada ${ }^{68}$. Une autre étape de cette transformation est l'instauration d'une politique de multiculturalisme par le gouvernement de Pierre Elliott Trudeau, le 8 octobre 1971.

Dans une lettre adressée à Robert Bourassa, Trudeau affiche sa volonté de construire une nation canadienne unie grâce à l'imposition, par l'État fédéral, d'un cadre normatif global. «Nous voulons reconnaître pleinement le fait, annonce-t-il, que le Canada est un pays multiculturel.» Ainsi, «la reconnaissance de tous les groupes ethniques et des différentes cultures à l'enrichissement de la vie canadienne contribuera, à notre avis, à renforcer chez tous nos concitoyens le sens de leur identité et le sentiment de leur appartenance nationale». «Tous les groupes culturels se sentiront mieux engagés dans l'œuvre commune et, conclut-il, l'unité canadienne en sera mieux raffermie ${ }^{69} .{ }^{.}$ Cette politique conteste ainsi toute prétention du Québec à une distinction fondée sur la culture.

D'emblée, les responsables politiques québécois soulèvent des réserves sérieuses, car la politique fédérale conteste la légitimité de leurs représentations de la $\mathrm{Cité}^{70}$. En réponse à Trudeau, Bourassa oppose le principe de l'égalité entre les deux peuples fondateurs. À cet égard, le multiculturalisme «paraît difficilement compatible avec la réalité québécoise où il y a une présence dominante de langue et de culture françaises, en plus d'une minorité importante de langue et de culture anglaises, ainsi que de nombreuses minorités d'autres langues

68. Raymond Breton, «The Production and Allocation of Symbolic Resources: an Analysis of the Linguistic and Ethnocultural Fields in Canada», Canadian Review of Sociology and Anthropology, 21,2 (1984): 129; Audrey Kobayashi, «Multiculturalism: Representing a Canadian Institution", dans James Duncan et David Ley, dir., Place/Culture/Representation (Londres, Routledge, 1993), 215-224.

69. E 47, art. 16, 9.12, Pierre Elliott Trudeau à Robert Bourassa, Ottawa, 7 octobre 1971, 1-2.

70. Fernand Harvey, «L'ouverture du Québec au multiculturalisme, 1900-1981», Études canadiennes, 12,2 (1986): 219-228 et «Les communautés culturelles et le multiculturalisme: une comparaison des politiques québécoise et canadienne», dans Jean-Michel Lacroix et Fulvio Caccia, dir., Métamorphoses d'une utopie (Paris/Montréal, Presses de la Sorbonne nouvelle/ Triptyque, 1992), 159-174; Evelyn Kallen, «Multiculturalism: Ideology, Policy and Reality», Journal of Canadian Studies, 17,1 (1982): 51-63. 
et d'autres cultures». Sans «qu'il puisse y avoir la moindre discrimination envers les autres cultures», l'État québécois devrait «assumer le rôle de premier responsable sur son territoire de la permanence de la langue et de la culture françaises dans le contexte nord-américain». Ce faisant, «il entend continuer de le faire par tous les moyens à sa disposition», dont son ministère de l'Immigration qui «administre déjà depuis plusieurs années des programmes analogues ${ }^{71} \gg$.

Dès lors, la stratégie de la souveraineté culturelle affronte celle du multiculturalisme. Jugeant que «la culture canadienne n'existe pas», le ministre des Communications, Jean-Paul L'Allier, voit «au Canada, sur un même territoire mais dans des lieux géographiques nettement délimités, la cohabitation de deux nations ayant des racines culturelles différentes». Pour L'Allier, la responsabilité des politiques culturelles revient «aux gouvernements provinciaux», surtout «s'il arrive que sur un territoire donné, comme c'est le cas du Québec, on retrouve les éléments constituants d'une nation ${ }^{72}$ ». Même un modéré comme le ministre des Affaires intergouvernementales, Gérard-D. Lévesque, réaffirme la ligne de conduite de son gouvernement, qui «vise à assurer, sur le territoire du Québec, la prédominance permanente de la langue et de la culture françaises», d'où sa forte insistance pour que cette politique «soit constamment orientée dans le sens de l'intégration des groupes ethniques au milieu francophone du Québec ${ }^{73}{ }^{»}$. Enfin, selon Bienvenue, le multiculturalisme a «un impact considérable»sur «les politiques linguistiques du Québec». Aussi rappelle-t-il «la nécessité d'une coordination étroite sous peine d'annuler les efforts entrepris $^{74}$ ».

Ce débat permet de comprendre l'inaction des responsables politiques québécois dans l'affaire des immigrants haitiens. En heurtant de plein fouet l'économie générale du symbole et le cadre normatif de l'État québécois, la contestation du multiculturalisme prend un caractère prioritaire dans l'élaboration de l'ordre du jour politique. La politique fédérale du multiculturalisme menace la stratégie de la souveraineté culturelle et remet en cause les responsabilités particulières du Québec comme berceau de la nation canadienne-française. Com-

71. E 47, art. 16, 9.12, Robert Bourassa à Pierre Elliott Trudeau, Québec, 11 octobre 1971, 1-3; ANQQ, E 42, Fonds du ministère des Affaires intergouvernementales, art. 154, Robert Bourassa, Le principe du multiculturalisme m'oblige à apporter des réserves sérieuses (Québec, 16 novembre 1971), 3.

72. E 47, art. 16, 9.12, Jean-Paul L'Allier, Notes de conférence. Le multiculturalisme et l'éducation internationale (s.1., 22 octobre 1974), 1-4.

73. E 47, art. 16, 9.12, Gérard-D. Lévesque à John Munro, Québec, 28 février 1975, 3.

74. E 47, art. 4, 1.18, Bienvenue, Les politiques actuelles..., 14. 
paré à ce défi, un problème ponctuel, tel que celui des immigrants illégaux, semble perçu comme secondaire et, à ce titre, moins urgent pour les responsables politiques québécois.

\section{Altérité et pluralisme culturel}

Comme fait de société, la question du multiculturalisme renvoie à une autre dynamique, celle des relations entre la société civile québécoise et les immigrants au tournant des années 1960. Le contact avec l'Étranger relève d'abord de l'imaginaire. En se représentant l'Étranger, apparaissent, tel un reflet, les images de Soi, ses valeurs, ses normes, ses objectifs et ses stratégies. Dans une révision des références premières de la Cité politique, l'altérité délie les paroles dans un débat de société, celui du pluralisme.

$\mathrm{Au}$ cours de l'événement des ressortissants haïtiens, le débat du pluralisme ne débouche pas encore sur un projet politique. Devant l'altérité, l'État québécois élabore ses normes nationales d'inclusion. Cette remise en cause des références est longue et ardue. Dès le projet de loi 75 créant le ministère québécois de l'Immigration, les parlementaires s'affrontent sur la promotion de la culture québécoise. Devraiton mettre de l'avant le droit collectif et favoriser la promotion de la francité? $\mathrm{Ou}$, dans un souci des droits individuels, devrait-on prôner le pluralisme comme politique d'État? La loi 75 confie au Ministère la mission de veiller à la «conservation des coutumes ethniques», tout en restant évasive sur le sens à donner «au développement et au progrès de la Province», et aux caractéristiques du «milieu québécois ${ }^{75}$ ».

Au début de la décennie 1970, la Province associe l'élément de la langue au concept de culture, contrairement à l'État fédéral ${ }^{76}$. Malgré les références aux droits de l'immigrant, de nature contractuelle, à travers les lois et règlements de l'État québécois, le flou des normes et l'absence de consensus témoignent encore, de 1972 à 1974, d'une certaine persistance des représentations «culturalistes» de la nation. Ces dernières s'estompent dans les objectifs et les stratégies de l'État québécois avec l'adoption des chartes des droits de la personne en 1975 et de la langue française en 1977. Les représentations d'une communauté politique contractuelle et d'une nation élective devien363 .

75. Statuts de Québec 1968, 16 Élizabeth II, chap. 68, art. 3 et 4; Pâquet, op. cit., 358-

76. Éric Waddell, «L’État, la langue et la société: les vicissitudes du français au Québec et au Canada", dans Alan Cairns et Cynthia Williams, dir., Les dimensions politiques du sexe, de l'ethnie et de la langue au Canada (Ottawa, Commission royale d'enquête sur l'intégration économique, 1986), 103. 
nent progressivement prédominantes au sein de l'État provincial, qui reconnaît le droit à la différence culturelle tout en recherchant l'insertion et la participation des immigrants à la francisation de la vie collective des Québécois ${ }^{77}$. En 1981, tout en légitimant l'État nation et la préséance de ces représentations porteuses d'une identité collective, le ministère québécois des Communautés culturelles et de l'Immigration, avec l'énoncé de politique Autant de façons d'être Québécois, énonce le paradigme d'une culture de convergence, distincte en sol d'Amérique.

L'affaire des ressortissants haïtiens montre la tension de la rencontre avec l'Étranger et de la redéfinition de Soi. En conclusion d'un reportage présenté à Format 60, Pierre Nadeau s'interroge: «La société québécoise est-elle disposée à accepter de se transformer en société multiraciale?» Cette question se transforme en critique dans la bouche du député créditiste Fabien Roy. «Si on veut faire du Québec une société multiraciale», la Province devrait prendre «ses responsabilités de façon que cette immigration se fasse en fonction des meilleurs intérêts du Québec et des Québécois ${ }^{78}$ ». Le 4 décembre 1974, répliquant à Nadeau, Bienvenue commente sa décision de ne pas aller de l'avant dans le dossier des immigrants illégaux: «Et voilà, la bombe explose. On dira: "Le ministère de l'Immigration est raciste"». Le ministre plastronne pour s'en défendre: «Non, je ne me cacherai pas d'employer les mots noir, jaune, rouge ou blanc et n'éviterai pas de parler "race" même si la bonne conscience de certains s'en trouve froissée et même si d'autres préfèrent croire qu'il n'y a pas de problème racial présent ou latent dans notre société.» Pour se justifier, Bienvenue avance en exemple l'émigration hypothétique de 20 millions d'Hindous [sic] vers le Canada, qui modifierait «la fibre sociologique de notre société». Aussi, «une société qui accepte le multiracisme trop au-delà d'une certaine norme fera face à des tensions sociales dangereuses ${ }^{79} \gg$. En établissant cette frontière normalisatrice que les immigrants illégaux n'ont pas respectée, l'État, selon Bienvenue, réduirait le potentiel de troubles sociaux.

La faute des troubles ne retombe pas sur les seules épaules des immigrants. Une part de responsabilité repose aussi sur la société d'accueil. La rencontre avec l'Étranger peut l'inquiéter. L'État devrait donc «sensibiliser et informer la population sur l'apport culturel et économique de nos frères d'autres origines ${ }^{80}{ }$. Cependant, le ministère

77. Helly, op. cit., 14-15.

78. $D A N Q, 15,71,29$ octobre 1974, 2402.

79. E 47, art. 8, 3.2.7, Bienvenue, Extraits du discours..., 3-5.

80. E 47, art. 4, 1.18, Bienvenue, Les politiques actuelles..., 13; Le Journal de Montréal, 11 avril 1974, 20. 
perçoit des «attitudes globales de crainte des Québécois vis-à-vis de l'étranger», «séquelles de l'histoire d'une communauté menacée et repliée sur elle-même ${ }^{81}{ }$. Devant la perception de l'insécurité culturelle $^{82}$, les responsables politiques québécois se donnent donc une mission éducatrice, voire sanitaire, contre «ce virus pernicieux qu'on appelle "xénophobie" "83». D'un ton méprisant, Bienvenue assimile les immigrants à «des handicapés moraux», victimes de l' «attitude parfois raciste et discriminante des Québécois francophones ${ }^{84} \gg$.

À ces assertions, la réplique la plus cinglante vient de l'éditorialiste Jean-Claude Leclerc. «Ce n'est pas la population mais les autorités qui sont restées fermées aux réfugiés chiliens et haïtiens.» Jacques-Yvan Morin réfute toute allégation de xénophobie puisque, «dans ce cas concret, c'est le contraire qui se produit: le peuple québécois s'est ému du sort des Haïtiens», sans faire «de distinction de couleur, en l'occurrence». Selon lui, les Québécois se montreraient «unanimement» favorables «à ce que ces Haïtiens restent parmi nous et puissent contribuer à la création de leur nouvelle patrie ${ }^{85}$ ».

\section{«Réparer un oubli» de la loi sur la langue}

Au moment de l'affaire des immigrants haïtiens s'opère une polarisation du débat linguistique. La trêve établie depuis la loi 63 et la création de la commission Gendron s'achève. Déposant son rapport le 31 décembre 1972, la commission propose au gouvernement de faire du français la langue officielle du Québec, tout en accordant à l'anglais le statut de langue nationale. Avant de légiférer, Bourassa préfère déclencher, en octobre 1973, des élections qu'il remporte avec une majorité écrasante. Puis, ne pouvant plus reculer l'échéance, il confie à son ministre de l'Éducation, François Cloutier, le soin de préparer une loi sur la question. Le 22 mai 1974, Cloutier présente le projet de loi 22 qui proclame le français langue officielle. La tempête éclate, enflammant les passions à la fois chez les intervenants anglophones, allophones et francophones. Les uns condamnent la restriction du libre choix qui violerait leurs droits. Les autres dénoncent la timidité des mesures coercitives ${ }^{86}$.

81. Québec, Ministère de l'Immigration, Rapport annuel 1973-1974 (Québec, Éditeur officiel, 1974), 14.

82. Robert Burns, DANQ, 15,85 (26 novembre 1974), 2964-2965.

83. Le Journal de Montréal, 11 avril 1974, 20.

84. Montréal-Matin, 16 décembre 1974, 3; Le Devoir, 16 décembre 1974, 3 et 6.

85. Le Devoir, 18 décembre 1974, 4; DANQ, 15,85 (26 novembre 1974), 2979.

86. Québec, Commission d'enquête sur la situation de la langue française et sur les droits linguistiques au Québec, Rapport, livre I: La langue de travail (Québec, Éditeur officiel, décembre 1972), 305; Marc V. Levine, The Reconquest of Montreal (Philadelphie, Temple University Press, 1990), 96-107. 
Dans les stratégies de règlement de l'épineux problème linguistique, Bourassa fait appel à Bienvenue lors de la préparation du projet de loi 22, même si ce dernier est un opposant convaincu de la coercition, lui préférant l'incitation et le «climat de liberté et de libre$\operatorname{arbitre}^{87}{ }_{\gg}$. La contribution de Bienvenue prend la forme d'un mémoire présenté en février 1974 au Conseil des ministres. Pour lui, toute politique linguistique faisant du français la langue officielle, pourrait «avoir des conséquences sur l'ampleur du mouvement migratoire au Québec», l'immigrant étant par définition mobile. Aussi, «une telle politique linguistique - clarifiée et conforme aux réalités québécoises - exige en retour une rénovation [sic] en profondeur de la politique d'immigration du Québec ${ }^{88}$ ».

$\mathrm{Au}$ lendemain même de l'adoption de la loi 22, Bienvenue présente le projet de loi 46 , modifiant la loi du ministère de l'Immigration. Le ministre a un objectif explicite, soit celui de «réparer un oubli» de la législation ${ }^{89}$. Suivant les recommandations de restructuration avancées par la commission Gendron ${ }^{90}$, il attribue à son ministère les fonctions «d'informer, de recruter, de sélectionner les immigrants» et «de rendre possible» l'établissement de ces nouveaux citoyens au Québec, afin d'assurer «leur intégration harmonieuse au sein de la collectivité québécoise, et plus particulièrement de la majorité francophone ${ }^{91} \gg$.

Dans ce débat, l'État québécois exprime une préférence implicite: l'immigrant idéal est un francophone ${ }^{92}$. Il faut donc le chercher où il se trouve. En 1974, des représentants du ministère québécois participent aux missions canadiennes de recrutement au Maroc et en Afrique équatoriale. À défaut de parlants français, le Québec pourrait accueillir ceux qui peuvent le devenir. D'où la création au ministère de la catégorie des «francophonisables ${ }^{93}$ ».

La question de la langue constitue une trame importante traversant l'événement des immigrants illégaux haïtiens. Bienvenue use de l'ar-

87. DANQ, 13,44 (15 mai 1973), B-1514; E 47, art. 4, 1.18, Bienvenue, Les politiques actuelles..., 7.

88. E 47, art. 27, Bienvenue, Politique linguistique..., 6-7.

89. Le Devoir, $1^{\text {er }}$ août 1974, 1 et 6; Dimanche-Matin, 4 août 1974, 5; The Montreal Gazette, 5 août 1974, 1.

90. Québec, Commission d'enquête sur la situation de la langue française et sur les droits linguistiques au Québec, livre III, op. cit., 131.

91. Le Devoir, $1^{\text {er }}$ août 1974, 1 et 6; Dimanche-Matin, 4 août 1974, 5; The Montreal Gazette, 5 août 1974, 1.

92. DANQ 13,44 (15 mai 1973), B-1512; E 47, art. 4, 1.18, Bienvenue, Les politiques actuelles..., 6.

93. La Presse, 19 octobre 1974, 3. 
gument linguistique dès sa première intervention dans le dossier en mars 1973. Lors du débat sur la motion de censure, la révocation de «ces politiques universelles et généreuses» lui apparaît bénéfique, étant donné les tensions présentes. En effet, les règlements fédéraux se seraient avérés «une prime à l'immigration non francophone ${ }^{94}$ ». Tous ne partagent pas son avis. Devant le sort réservé aux immigrants illégaux, la violence des critiques témoigne des profonds soupçons de duplicité prêtés au gouvernement Bourassa. Pour Camil Samson, l'expulsion des Haïtiens relèverait d' «une attitude anti-immigration de langue française» et de la «peur» d'Ottawa face à l'augmentation du nombre des nouveaux venus francophones. Par «son silence, [l'État québécois] endosse cette cochonnerie qu'est en train de faire le gouvernement du Canada». Plus mesuré, Jacques-Yvan Morin n'en démolit pas moins la stratégie linguistique mise sur pied par Bienvenue. «Qu'attend-il pour exercer ces fameux pouvoirs» de recrutement, de sélection et d'assistance, tance le chef de l'opposition. «N'est-ce pas l'occasion rêvée? Ces gens sont déjà sur place, ils sont déjà en voie d'intégration, ce sont des francophones.» Le gouvernement Bourassa mérite donc le blâme, selon Morin ${ }^{95}$.

\section{Les droits de la personne}

Pendant l'événement des ressortissants haïtiens, les hommes politiques québécois s'accordent une responsabilité et des capacités limitées d'intervention, sur «le plan moral pour des raisons humanitaires». Dès ses premières démarches auprès d'Andras, Bienvenue adopte ce principe. "Accueillir un immigrant, le recevoir et l'aider à s'intégrer, ajoute-t-il plus tard, c'est faire preuve d'humanisme, puisqu'il s'agit d'un être humain, quels que soient sa race ou sa culture, sa couleur ou son origine, sa langue ou sa religion ${ }^{96} . »$ Dépassant la simple compassion, la dimension humanitaire renvoie à une conception de l'individu doté de droits et de libertés reconnus par la communauté politique.

À partir des années 1960, divers groupes se mobilisent afin d'exiger de la part des États la garantie des droits individuels et collectifs. La question s'inscrit dans une mutation de la culture politique internationale où la thématique des libertés fait florès dans les débats de la

94. E 47, art. 8, 3.2.7, Jean Bienvenue, Déclaration concernant la situation touchant certains Haïtiens (Québec, 29 octobre 1974), 2; DANQ, 15,71 (29 octobre 1974), 2398; 15,85 (26 novembre 1974), 2981-2982.

95. Samson, $D A N Q, 15,78$ (8 novembre 1974), 2703; 15,85 (26 novembre 1974), 2957 et 2962. Morin, ibid., 2979.

96. E 47, art. 4, 1.18, Bienvenue, Les politiques actuelles..., 15; DANQ, 15,71 (29 octobre 1974), 2398. 
critique du totalitarisme, de la dissociation progressive entre l'État administratif et l'intérêt du public, ainsi que de la promotion des droits de la personne ${ }^{97}$. Avec la Révolution tranquille, l'appareil étatique québécois s'impose une série de principes axiologiques universels et légitimes, tels les droits et les libertés démocratiques, afin d'assurer une régulation plus efficace des relations entre l'Etat et les citoyens, entre autres dans sa gestion de l'inclusion ${ }^{98}$. Dès novembre 1972, Québec songe à se doter d'une charte des droits de la personne. L'État québécois passe aux actes en octobre 1974 avec le dépôt du projet de loi 50, visant à subordonner le champ des compétences québécoises aux principes directeurs des droits et des libertés. L'Assemblée nationale débat de ce projet de loi en pleine polémique des ressortissants haïtiens. Témoignant du consensus existant sur le projet de société démocratique, les parlementaires adoptent à l'unanimité la Charte québécoise des droits et libertés le 27 juin 1975. Entre autres garanties, la Charte reconnaît des droits collectifs aux minorités ethniques issues de l'immigration. Le débat sur les droits de la personne a donc des incidences sur l'événement des immigrants illégaux.

Débutons par le volet axiologique. Pour les critiques du gouvernement, l'affaire constitue un cas d'espèce où les valeurs de l'État de droit doivent prévaloir, particulièrement en ce qui concerne la vie et la sécurité des individus. Camil Samson s'en fait le héraut à l'Assemblée. Pour lui, «il y va de la vie de ces gens-là». «S'étant exprimés librement sur notre territoire», les ressortissants haïtiens «risquent, au retour», des «représailles qui peuvent aller même jusqu'à la peine de mort». Pour appuyer sa thèse, il cite les dispositions de la «loi anticommuniste d'Haïti» du 28 avril 1969, punissant de mort les fautifs. Rappelant la crise d'octobre, Samson argue de l'autorité des articles du projet de charte des droits et libertés. «En vertu de ce principe que toute personne dont la vie est en péril a droit au secours, nous avons le devoir, en tant que Québécois, en tant que Canadiens français, de nous lever et de dire: présents.» La critique de Samson se fait alors virulente. «Le gouvernement québécois s'apprête à violer, par son inaction, les grands principes qu'il a lui-même placés dans la Charte québécoise des droits de l'homme ${ }^{99} . » I c i$, Bienvenue temporise: «Il

97. Laurent Cohen-Tanugi, La métamorphose de la démocratie française (Paris, Gallimard, 1993), 31.

98. Louise Fontaine, L'organisation étatique de l'inclusion et de l'exclusion: le cas du Québec (1976-1988), thèse de doctorat (science politique), Université Laval, 1990, 46-74; Helly, op. cit., 33-180; Pâquet, op. cit., 381-394.

99. $D A N Q, 15,78$ (8 novembre 1974), 2702; 15,85 (26 novembre 1974), 2958, 29602961, 2963 et 2991. 
faut s'entourer de la plus grande prudence lorsqu'on parle de cette question [fort] complexe ${ }^{100} . »$

Un autre volet de la dimension humanitaire touche l'aspect normatif et régulateur. L'adoption d'une charte québécoise des droits et libertés va dans ce sens. Un État de droit doit prévoir des mécanismes d'inclusion à la communauté politique, avec des catégories préconçues, aux attributs normatifs et communs à tous ses membres. Ainsi catégorisés, les individus acquièrent une identité sociale ${ }^{101}$. Dès lors, l'État peut exercer son pouvoir légitime en régulant les situations problématiques, réduisant d'autant les déviances. L'affaire des ressortissants haïtiens se présente d'ailleurs sous cet angle. Depuis novembre 1972, Ottawa opère une première catégorisation en identifiant les visiteurs avec un visa de touriste demandant la citoyenneté canadienne. Puis le fédéral normalise en retirant son règlement.

Les autorités fédérales font une seconde catégorisation. En se référant à la convention des Nations unies sur le statut des réfugiés, le ministère fédéral considère que ce statut est décidé en fonction de l'individu, et non par rapport à un groupe. Andras fait ici une distinction majeure. Certains Haïtiens seraient des réfugiés politiques, mais «la majorité» n'aurait «pas été persécutée dans leur propre pays» et n'essayerait «de parvenir à rester» au Canada «que pour des raisons économiques ${ }^{102}{ }$.

Le préjugé d'Andras choque. Robert Burns rappelle, outré, les propos du secrétaire d'État haïtien Pierre Gousse, qui avait affirmé que les ressortissants de son pays présents au Québec n'étaient «rien de moins que des éléments subversifs». "Alors imaginez, renchérit Burns, quelle réception se préparent ces Haïtiens qu'on va retourner chez eux». Enfin, le ministre Bienvenue «a tort d'endosser [un] gouvernement fédéral inefficace, bêtement légaliste, [voire] totalement immoral devant le problème des Haïtiens ${ }^{103}$ ».

Toutefois, la position du gouvernement Bourassa reconnaît la légitimité de la mesure fédérale qui exprime le pouvoir normatif de l'Etat. Le ministère de l'Immigration juge qu'un grand nombre de politiques gouvernementales sont, par nature, discriminatoires sans être condamnables. Au contraire, elles traduisent la volonté d'imprimer une direction aux événements. Plus encore, la raison d'État peut

100. DANQ, 15,78 (8 novembre 1974), 2706.

101. Erving Goffman, Stigma. Notes on the Management of Spoiled Identity (Englewood Cliffs, NJ, Prentice-Hall, 1963), 2.

102. Cité par Bienvenue, DANQ, 15,71 (29 octobre 1974), 2399.

103. DANQ, 15,71 (29 octobre 1974), 2400-2401; 15,85 (26 novembre 1974), 2964. 
s'avérer un outil utile dans la poursuite de ses objectifs. Aussi, le Québec «ne veut pas critiquer le pouvoir discrétionnaire parce qu'on peut l'orienter en fonction de l'immigration francophone ou francophonisable ${ }^{104} \gg$. Les cognoscentis du ministère de même que les députés libéraux endossent la catégorisation et la sélection discriminatoire de l'État canadien. Au moment du débat sur la motion de censure, Bienvenue consacre presque tout son temps de parole à présenter longuement les objectifs et les stratégies employés par l'État fédéral dans cette affaire, pour en souligner toute la légitimité ${ }^{105}$.

$$
* * *
$$

De novembre 1972 à décembre 1974, l'affaire des immigrants illégaux haïtiens secoue l'échiquier politique québécois. Une secousse de faible amplitude, dira-t-on, mais révélatrice des mouvements tectoniques, des forces souterraines, des multiples tendances complexes du changement social qui s'animent sous l'écorce du récit historique. Le sismographe de l'historien en capte peut-être faiblement les ondes, mais, par le jeu des échelles d'observation, il peut mieux saisir les sens hétérogènes de cette configuration temporelle.

Appréhender la complexité du sens d'un événement oblige ainsi le praticien de l'histoire à réfléchir sur des gestes du métier, soit ceux de la lecture et de l'écriture. Tout d'abord, lire le matériau historique, ces poussières d'information dispersées parmi des sources diverses, impliquerait dorénavant non pas la recherche d'une représentativité simplificatrice dans la construction du fait historique, mais plutôt la quête et l'interprétation d'indices porteurs de sens et révélateurs du changement social. Une telle lecture herméneutique déboucherait sur une révision des catégories analytiques traditionnelles de la durée, comme celles des dynamiques structurelles et conjoncturelles. De plus, elle inciterait l'historien à user plus souvent des variations d'échelle, de la perspective microhistorique à l'analyse des tendances macrosociales, selon l'objet étudié et la documentation y afférant.

Ensuite, écrire le fait historique, le construire en présentant les diverses interprétations de son sens, le configurer sous la forme d'un récit, exigerait de rendre compte de sa complexité inhérente. Tout en étant soucieux d'une certaine cohérence du sens, il serait possible de concevoir d'autres modes d'organisation du récit, structurés autour des acteurs et de leurs relations, ou suivant le jeu des tendances

104. E 47, art. 3, 1.11.1, Québec, Ministère de l'Immigration, La position..., 36 et 41; art. 2, 1.7.1, Bienvenue, Conférence de presse..., 8-12.

105. DANQ, 15,85 (26 novembre 1974), 2981-2989. 
sociohistoriques. Plus fidèles au réel, ces récits deviendraient ainsi moins linéaires et simplificateurs que ceux modelés par les canons de la narration ou des problématiques de la causalité. Une autre attitude de lecture, des modes différents d'écriture: voilà autant de défis, soulevés par l'étude de ce cas, qui se présentent désormais à l'historien ${ }^{106}$.

106. En plus des considérations empiriques se dégageant de cette étude de cas, il serait possible d'envisager une réflexion théorique autour de la complexité de l'événement, des nouveaux sens donnés aux notions de structure et de conjoncture, ainsi que de leur utilisation éventuelle dans les sciences humaines, tout particulièrement dans la discipline historique. C'est un défi que nous entendons relever prochainement. 